\title{
Real Time Video Enhancement using Camera Response Model
}

\author{
Ch.Sharvani ${ }^{1}$, D. Praveen Kumar $^{2}$, P. Bhavya ${ }^{3}$, P. Sri Vaishnavi ${ }^{4}$ \\ ${ }^{1}$ UG Student, Department of Electronics and Communication Engineering, Vardhaman College of Engineering, \\ Hyderabad, Telangana, India, chsharvani.vce@gmail.com \\ ${ }^{2}$ Assistant Professor, Department of Electronics and Communication Engineering, Vardhaman College of \\ Engineering, Hyderabad, Telangana, India, praveenkumar7.d@gmail.com \\ ${ }^{3}$ UG Student, Department of Electronics and Communication Engineering, Vardhaman College of Engineering, \\ Hyderabad, Telangana, India, bhavyapolu.16@gmail.com \\ ${ }^{4}$ UG Student, Department of Electronics and Communication Engineering, Vardhaman College of Engineering, \\ Hyderabad, Telangana, India, srivaishnavipusuluri7@gmail.com
}

\begin{abstract}
In this paper, we propose a method to enhance the quality of real time video based on Camera Response Function. Most of the existing enhancement algorithms introduce some distortion because of ignoring non linear camera process. We propose a method by taking camera response function into consideration to provide acceptable video quality. The performance of this approach is demonstrated through experimental results and measurements. Experimental results show that the proposed approach reduce the computational time cost about $60 \%$ compared with the three channel camera response curve with acceptable distortions.
\end{abstract}

Key words : Real Time Video Enhancement, camera response function (CRF), Brightness Transform Function (BTF), Low light video enhancement.

\section{INTRODUCTION}

Videos are the integral part of our life as they are used in many sectors such as schools, hospitals, companies' airports etc. Capturing a video in low light intensity places i.e. in dark conditions is still being a drawback. When the image/video was taken for investigation, it needs to be clean for possible effective results. Modern digital cameras are still limited in capturing high-dynamic range. Videos in low-light conditions and the longer exposure time in the camera often results in motion blur [1].

In order to remove the motion blur and to provide clear visual quality, video enhancement method is used and enhancing a video is really a tedious job. Enhancement of the video is improving the visual quality of a video. Various algorithms or approaches are available for enhancement. The basic methods are improvising properties of videos such as color, contrast and brightness. Noise suppression and noise muting methods are mostly used in enriching the videos. In present situation of the world, there are millions cameras are being actively working which are used for CCTVs, video conferencing, production of videos (movies/short films) and etc.

Due to the increasing demand of CCTV cameras, cost effectives CCTV cameras are being manufactured and used. Since the CCTV cameras are of low cost, there are some limitations such as: lack of sharp sensors; lack of sophisticated video enhancing processors; collective graphics processing chips. Even though these cameras are not good at capturing HD videos, these are widely used. In many applications, these CCTVs had to be monitored in real time. For example, in banking sector, surveillance cameras are used to protect from robberies. If the cameras are not providing a quality video, it will become hard to monitor and observer of CCTV cameras may miss a compulsory scene, which result in huge damage.

Recently, researchers have been proposed various algorithms to address low illumination problems. Some of the basic approaches are histogram equalization [2] - [5], which increases the global contrast of the image. This process distributes the intensity values over the histogram. In [6] [8], authors have proposed an approach Gamma correction for illumination problem. Because of using global information, these approaches are suffers from intensity saturation which results inconsistent enhancement. To overcome over enhancement, advanced algorithms like adaptive histogram equalization [9] - [10] and retinex algorithm [11] -[14] have been proposed by considering the pixel values and global information together.

In [15] and [16], authors have proposed algorithms to enhance and de-noise low-brightness images by taking image noise into consideration. In [17], authors have been proposed an approach to increase the contrast of satellite images using lifting wavelets and singular Value Decomposition (SVD). In [18] - [19], authors solve the low illumination problem by using de-hazing techniques. But these algorithms are suffers from high complexity order, hence these are not preferred for 
real time applications. However, aforementioned approaches are ignoring the non linear camera process Camera Response Function (CRF), these approaches may introduce distortion to the enhanced image/video.

To solve above problem, real time video enhancement approach is presented by considering non-liner camera process (CRF). Our work is divided into:

1. Using different exposure ratio images estimate the camera response model and determine its parameters.

2. Obtain the camera response curves for the three channels (Red, Green and Blue).

3. We prove that approximated fixed camera response curve obtain enhanced result with some tolerable distortion compared with three channel camera response curve

The remainder of the paper organized as follows: Overview of camera response model is presented in section 2. In section 3 we presented our enhancement approach, hardware implementation results are presented in section 4. And finally we concluded our paper in section 5 .

\section{BACKGROUND}

The excessive need of night operations requires more elaborated and collective information from the enhanced image/video. However, most of the CCTV cameras lack the ease to identify and understand the situation due to their inability of covering nearby location and their conditions because of substandard illumination. Hence, improvisation of video quality is necessary and difficult element of CCTV surveillance system.

Most of the camera manufacturing companies incorporate white balancing operation (which is non-linear) in camera processes to improve the quality of the image/video. These non linear processes are ignoring by the algorithms which are used to determine irradiance and illumination, leads to performance degradation. Therefore, Camera Response Function (CRF) has been proposed to model such non linear camera processes, it describes the relation between image pixel values $Z$ and irradiance $E$.

$$
Z=h(E)
$$

Where $h()$ is the non linear Camera response function. But the change in the exposure settings of the camera leads to linear change in the irradiance. Since $h()$ is the non-linear function, the image pixel values may not change linearly with respect to image irradiance. Therefore, a non linear mapping function, called as Brightness Transfer Function is used to map the various exposure images, the BTF for the two images $Z_{1}$ and $Z_{2}$ captured with the same camera with different exposures, is given by

$$
Z_{2}=f\left(Z_{1}, k\right)
$$

From the definitions of CRF and BTF, these two non linear functions are related as [20]

$$
f(h(E), k)=h(k E)
$$

From the traditional model, irradiance can be decomposed by reflectance $(R)$ and illumination components ( $I$ ). According to this, the input image can be written as

$$
Z=h(R \square I)
$$

where $\square$ is the element-by-element multiplication. The enhanced image can be written as [20]

$$
Z_{\text {enh }}=f(Z, 1 \% \mathrm{I})=f(Z, k)
$$

Where $k$ is the exposure ratio matrix, which indicates the exposure ratio of each pixel in the image. Exposure is the amount of light which reaches your camera sensor or film. Since $k$ is inversely proportional to illumination component $I$, lightning of the video is increased by reducing the exposure ratio map. And it is given by

$$
k=1 \% \mathrm{I}
$$

Where $\%$ is the element-by-element division. From the above analysis, video enhancement includes determination of CRF and its parameters and then estimation of exposure ratio matrix.

\section{CAMERA RESPONSE MODEL}

Most of the existing enhancement approaches ignoring the camera response characteristics, which leads to under and/or over enhancement. In this paper, we consider the response model of the camera which is used in the hardware implementation. Using different exposure ratio images from the camera (webcam) estimate the camera response model characteristics and its parameters. Finally, we enhance the video (images streams) using equation (5)

Camera response model determine the camera response function and BTF. CRF is depends on the camera used, which gives the characteristic of the cameras. Every camera has a unique camera response curve for each channel (Red, Green, and Blue). Whereas, BTF is depends on the camera and exposure ratio. BTF function can be obtained by using two images from the same camera with different exposure ratios. In [21], the authors have proposed a two parameter camera response model; Sigmoid, based on human vision system. The CRF and BTF of Sigmoid model are given by:

$$
\begin{aligned}
& h(E)=(1+u) \frac{E^{v}}{E^{v}+u} \\
& f(Z, k)=\frac{k^{v} Z(1+u)}{\left(k^{v}-1\right) Z+1+u}
\end{aligned}
$$
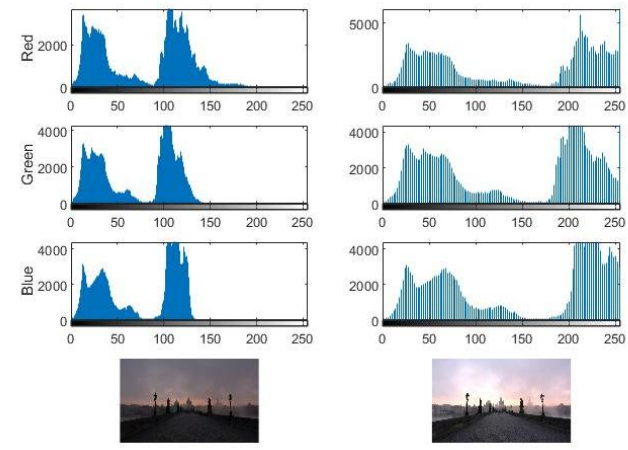

Figure 1: Observation of Sigmoid Camera Response Model 
Table 1: Commonly used Camera Response Models

\begin{tabular}{|c|c|c|}
\hline Model & $\begin{array}{c}\text { Camera Response Function } \\
\text { (CRF) } h(\mathrm{E})\end{array}$ & $\begin{array}{c}\text { Brightness Transform } \\
\text { Function (BTF) } f(\square, k)\end{array}$ \\
\hline Preferred Correction & $\left(\frac{e^{v} E^{u}}{e^{v} E^{u}+1}\right)^{t}$ & $\left(\frac{Z^{1 / t} k^{u}}{Z^{1 / t}\left(k^{u}-1\right)+1}\right)^{t}$ \\
\hline Gamma Correction & $e^{E^{u}}$ & $Z^{k^{u}}$ \\
\hline Sigmoid & $(1+u) \frac{E^{v}}{E^{v}+u}$ & $\frac{k^{v} Z(1+u)}{\left(k^{v}-1\right) Z+1+u}$ \\
\hline $\begin{array}{l}\text { LIME } \\
\text { Beta-Gamma }\end{array}$ & $\begin{array}{l}E^{t} \\
\begin{cases}\exp \left(v\left(1-E^{u}\right)\right) & \text { if } \gamma \neq 1 \\
E^{t} & \text { if } \gamma=1\end{cases} \end{array}$ & $\begin{array}{l}k^{t} Z \\
\begin{cases}\exp \left(v\left(1-k^{u}\right) Z^{k^{u}}\right. & \text { if } u \neq 0 \\
k^{t} Z & \text { if } a=0\end{cases} \end{array}$ \\
\hline
\end{tabular}

Figure 1 shows the performance of sigmoid function along with the histograms of three planes. Observe that the histograms of low light images concentrated in low-brightness area. After the sigmoid model, histograms of three plans are distributed to the entire range.

For low light image enhancement authors in [21], has presented a two parameters Beta-Gamma camera response model. The BTF of Beta-Gamma model is given by:

$$
f(Z, k)=\beta Z^{\gamma}
$$

Beta and Gamma are the exposure related parameters. The closed form of camera response function is presented in [20], as follows:

$$
h(E)= \begin{cases}\exp \left(v\left(1-E^{u}\right)\right) & \text { if } \gamma \neq 1 \\ E^{t} & \text { if } \gamma=1\end{cases}
$$

Where $u, v$ and $t$ are model parameters. $u=\log _{k} \gamma, v=\frac{\ln \beta}{1-\gamma}$ and $t=\log _{k} \beta$.

After the determination of camera response model, its parameters are evaluated for every channel i.e., red, green and blue. Calculation of response curve for three channels for every frame of the video, leads to high processing time. But real time video applications are less sensitive to the time. Therefore instead of calculating three channels curve, average response curve $f s$ is approximated. Table 1 shows the some of the commonly used camera response models.

\section{RESULTS AND DISCUSSIONS}

In this section, we presented our implantation results. Our work is implemented on arduino uno board and simulated using MATLAB. The program was tested on Intel $®$ core TM i3-6006U @ 2.00GHz with 4GB RAM. We captured the video in real time using camera, through the arduino captured video is transferred to the MATLAB in serial for enhancement.

Figure 2 shows the comparison of performance of response model with three channel curve and fixed approximated response curve. From the results we observe that the approximated camera response curve introduce acceptable distortion as compared with the three channel response curve.

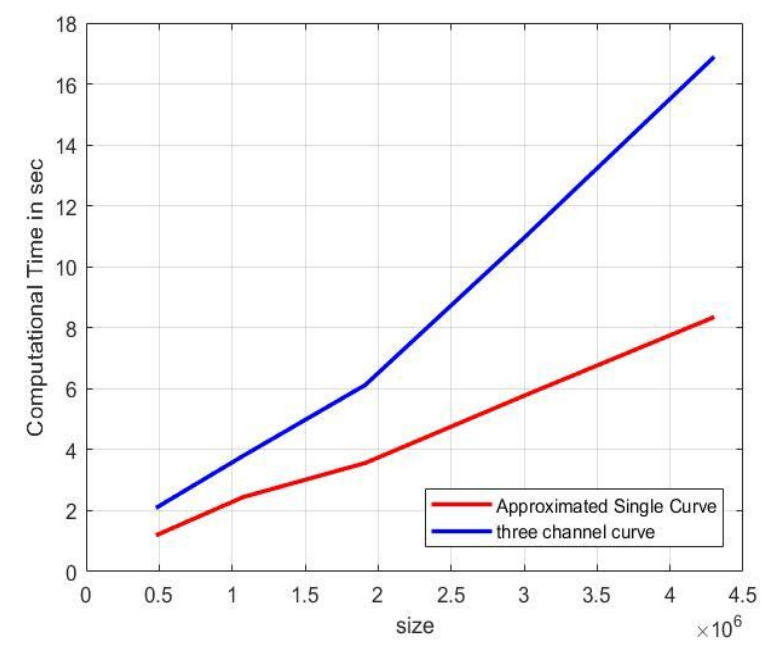

Figure 3: Computational Time comparison

Computational time cost of two approaches is presented in figure 3. However, approximated fixed camera response curve introduce some tolerable distortion, it is quite time consuming approach. Since our approach considers approximated curve for the three channels, there is reduction of parameter estimation of three channels computations for every frame, and results in reduction in computational time cost. From the results we observe that the, our approach reduces computation time cost about $60 \%$ with acceptable distortion. 


\section{INPUT}
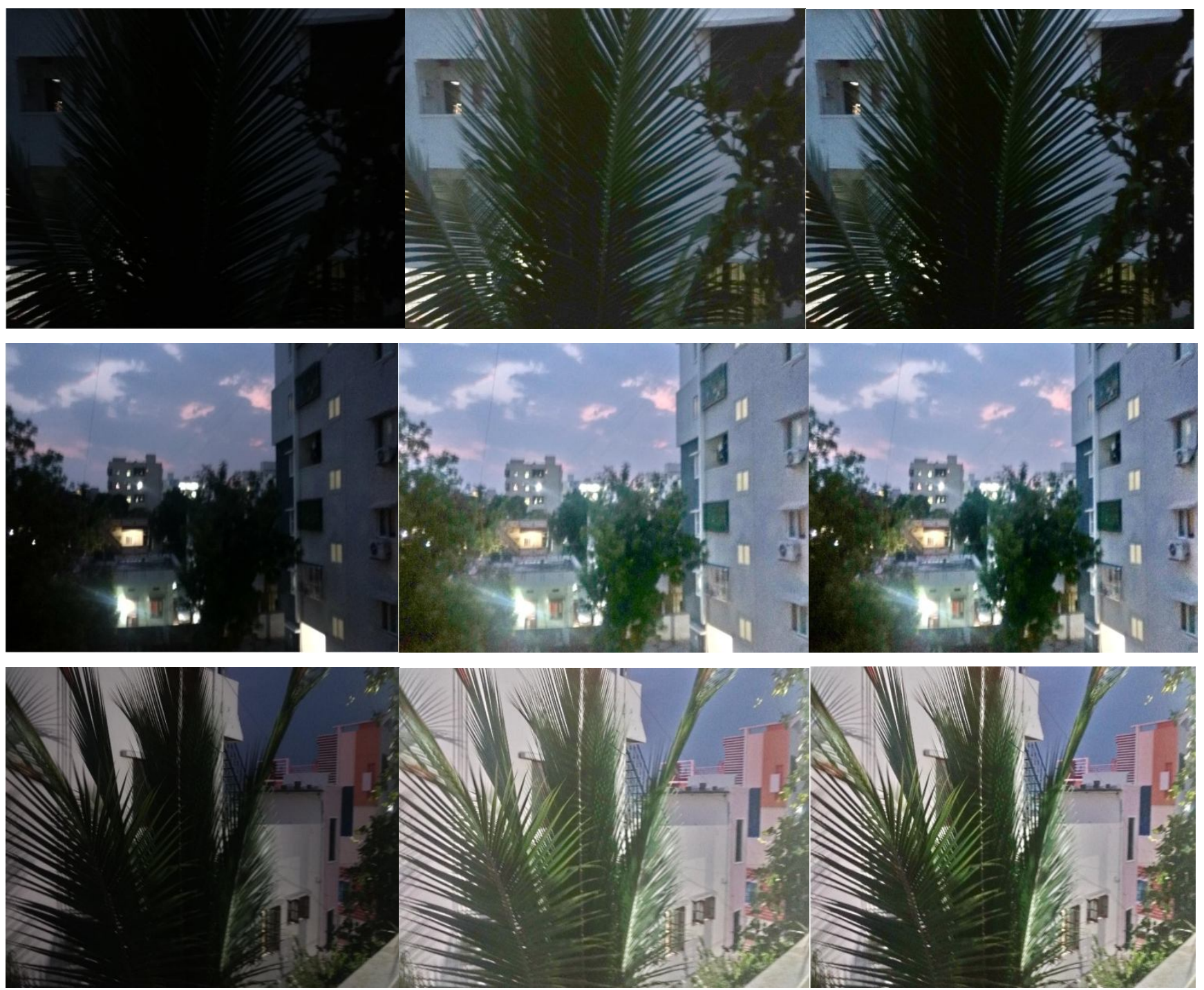

Figure 2: Screenshots from the videos before and after (using three channel curve and single approximated curve) the enhancement

\section{CONCLUSION}

In this paper we proposed a video enhancement approach by considering a non linear Camera Response Function and retinex model to adjust the exposure of low light video frames. For every camera, we need to evaluate the camera response curve for three channels i.e., Red, Green and Blue. Exposure and response model parameters are estimated and used in enhancement. To reduce the computational time, we approximated the fixed camera response curve. From the simulation results we conclude that the proposed approach gives more video quality (with tolerable distortions) with less computational time cost. Our approach reduces the time cost around $60 \%$ (approx.) by compared with the three channel camera response curves.

\section{REFERENCES}

1. Chi-Yi Tsai, A Fast Dynamic Range Compression With Local Contrast Preservation Algorithm and Its
Application to Real-Time Video Enhancement, IEEE Transactions on Multimedia, Volume: 14, Issue: 4 , Aug. 2012. https://doi.org/10.1109/TMM.2012.2190390

2. Y.-T. Kim, Contrast enhancement using brightness preserving bihistogram equalization, IEEE Trans. Consum. Electron., vol. 43, no. 1, pp. 1-8, Feb. 1997.

3. Y. Wang, Q. Chen, and B. Zhang, Image enhancement based on equal area dualistic sub-image histogram equalization method, IEEE Trans. Consum. Electron., vol. 45, no. 1, pp. 68-75, Feb. 1999.

https://doi.org/10.1109/30.754419

4. T. Arici, S. Dikbas, and Y. Altunbasak, A histogram modification framework and its application for image contrast enhancement, IEEE Trans. Image Process., vol. 18, no. 9, pp. 1921-1935, Sep. 2009. https://doi.org/10.1109/TIP.2009.2021548

5. S. Battio, A. Castorina, and M. Mancuso, High dynamic range imaging for digital still camera: An overview, 
Journal Electron. Imag., vol. 12, no. 3, pp. 459-469, Jul. 2003.

https://doi.org/10.1117/1.1580829

6. N. Moroney, Local color correction using non-linear masking, in Proc. 8th Color Imag. Conf., Nov. 2000, pp. $108-111$.

7. X. Guan, S. Jian, P. Hongda, Z. Zhiguo, and G. Haibin, An image enhancement method based on gamma correction, in Proc. IEEE Int. Symp. Comput. Intell. Design, Dec. 2009, pp. 60-63.

8. H. Farid, Blind inverse gamma correction, IEEE Trans. Image Process., vol. 10, no. 10, pp. 1428-1433, Oct. 2001. https://doi.org/10.1109/83.951529

9. S.-C. Huang, F.-C. Cheng, and Y.-S. Chiu, Efficient contrast enhancement using adaptive gamma correction with weighting distribution, IEEE Trans. Image Process., vol. 22, no. 3, pp. 1032-1041, Mar. 2013.

10. D. Zhang, W. J. Park, S. J. Lee, K. A. Choi, and S. J. Ko, Histogram partition based gamma correction for image contrast enhancement, in Proc. IEEE 16th Int. Symp. Consum. Electron. (ISCE), Jun. 2012, pp. 1-4. https://doi.org/10.1109/ISCE.2012.6241687

11. Z.-U. Rahman, D. J. Jobson, and G. A. Woodell, Retinex processing for automatic image enhancement, $J$. Electron. Imag., vol. 13, no. 1, pp. 100-110, 2004.

12. C.-T. Shen and W.-L. Hwang, Color image enhancement using Retinex with robust envelope, in Proc. IEEE Int. Conf. Image Process., Nov. 2009, pp. 3141-3144.

13. B. Li, S. Wang, and Y. Geng, Image enhancement based on Retinex and lightness decomposition, in Proc. IEEE Int. Conf. Image Process., Sep. 2011, pp. 3417-3420.

14. M. K. Ng and W. Wang, A total variation model for Retinex, SIAM J. Imag. Sci., vol. 4, no. 1, pp. 345-365, 2011.

https://doi.org/10.1137/100806588
15. A. Łoza, D. R. Bull, P. R. Hill, and A. M. Achim, Automatic contrast enhancement of low-light images based on local statistics of wavelet coefficients, Digit. Signal Process., vol. 23, no. 6, pp. 1856-1866, Dec. 2013.

16. C. Jung, Q. Yang, T. Sun, Q. Fu, and H. Song, Low light image enhancement with dual-tree complex wavelet transform, J. Vis. Commun. Image Represent., vol. 42, pp. 28-36, Jan. 2017.

17. Dr. P. M. K. Prasad, Dr. Y. Raghavender Rao, Dr. Karri Chiranjeevi, Contrast Enhancement Of Satellite Images Based on Lifting Haar Wavelet Transform And Singular Value Decomposition, International Journal of Emerging Trends in Engineering Research, vol. 8, n0. 3, pp. 830-837, March 2020. https://doi.org/10.30534/ijeter/2020/37832020

18. X. Dong et al., Fast efficient algorithm for enhancement of low lighting video, in Proc. IEEE Int. Conf. Multimedia Expo, Jul. 2011, pp. 1-6.

19. L. Li, R. Wang, W. Wang, and W. Gao, A low-light image enhancement method for both denoising and contrast enlarging, in Proc. IEEE Int. Conf. Image Process. (ICIP), Sep. 2015, pp. 3730-3734.

20. Yurui Ren, Zhenqiang Ying, Thomas H. Li, and Ge Li, LECARM: Low-Light Image Enhancement Using the Camera Response Model, IEEE Transactions on Circuits And Systems for Video Technology, vol. 29, no. 4, April 2019. https://doi.org/10.1109/TCSVT.2018.2828141

21. S. Mann. Comparametric equations with practical applications in quanti-graphic image processing, IEEE transactions on image processing, 9(8):1389-1406, 2000.

https://doi.org/10.1109/83.855434 